\title{
Update on Lorlatinib: Role in Reducing the Risk of Disease Progression in ALK-Positive NSCLC
}

\author{
Karen M Yun (D), Lyudmila A Bazhenova \\ Department of Hematology-Oncology, Moores Cancer Center at UC San Diego Health, La Jolla, CA, USA \\ Correspondence: Lyudmila A Bazhenova, Department of Hematology-Oncology, Moores Cancer Center at UC San Diego Health, 3855 Health \\ Sciences Drive, La Jolla, CA, 92093, USA, Tel + I 858-822-6I89, Fax + I 858-822-6I90, Email Ibazhenova@health.ucsd.edu
}

\begin{abstract}
Lorlatinib is an oral third-generation inhibitor of anaplastic lymphoma kinase (ALK) with activity in advanced ALKpositive non-small cell lung cancer (NSCLC) in both the first and subsequent line setting. Superior systemic and intracranial efficacy of lorlatinib over crizotinib, a first-generation ALK tyrosine kinase inhibitor (TKI), in treatment-naïve patients with advanced ALKpositive NSCLC was demonstrated by the phase 3 CROWN trial. Lorlatinib retains anti-tumor effect against single and some compound ALK resistance mutations after disease progression on first- and second-generation ALK TKIs. Currently, alectinib, brigatinib, ceritinib, crizotinib and lorlatinib are approved for treatment of advanced ALK-positive NSCLC. However, no head-tohead studies have directly compared lorlatinib to second-generation ALK inhibitors. Herein, we aim to provide an overview of the efficacy and safety of lorlatinib and discuss where lorlatinib stands in the therapeutic approach to advanced ALK-positive NSCLC. Keywords: anaplastic lymphoma kinase, lorlatinib, non-small cell lung cancer
\end{abstract}

\section{Introduction}

Advancements in our understanding of the molecular biology of non-small cell lung cancer (NSCLC) have made targeted therapies possible for patients with oncogene-driven lung cancers. Anaplastic lymphoma kinase (ALK) gene rearrangements are present in about 5\% of patients with NSCLC and mainly occur in younger patients with a never or light smoking history and adenocarcinoma histology. ${ }^{1}$ ALK rearrangements were first described in NSCLC by Soda et al, who identified the EML4-ALK fusion transcript in NSCLC cells. Using a retroviral cDNA expression system generated from a lung adenocarcinoma specimen, Soda et al isolated the EML4-ALK fusion gene in mouse fibroblasts with transformed foci in cultures and expression of EML4-ALK plasmid clones. ${ }^{2}$ Additional variants of EML4-ALK due to differences in breakpoints as well as translocations of ALK with other partner genes have been described in NSCLC. ${ }^{3}$

Discovery of the oncogenic ALK fusion gene paved the way for therapeutic targets against ALK in advanced NSCLC. Crizotinib, a first-generation inhibitor of ALK, demonstrated superior efficacy over chemotherapy in phase 3 clinical trials (PROFILE 1007 and PROFILE 1014) and was the first ALK targeted drug approved by the Food and Drug Administration (FDA) in the frontline treatment of metastatic ALK-positive NSCLC. ${ }^{4,5}$ However, a majority of patients develop resistance to crizotinib within 12 months on therapy. Central nervous system (CNS) penetration by crizotinib is poor, with CNS progression observed in $70 \%$ of patients with known brain metastases. ${ }^{5,6}$ Second-generation ALK inhibitors (alectinib, brigatinib, ceritinib, ensartinib) made headway after the approval of crizotinib, demonstrating higher potency and superior intracranial responses in advanced ALK-positive NSCLC than crizotinib. ${ }^{7-10}$

Lorlatinib, an oral third-generation inhibitor of ALK and c-ros oncogene 1 (ROS1) kinases, initially received accelerated FDA approval in 2018 as subsequent line therapy of metastatic ALK-positive NSCLC and is the latest ALK inhibitor to be granted approval for use in the first-line setting. ${ }^{11-13}$ Lorlatinib was developed from crizotinib using a structure-based drug design approach. Its macrocyclic structure distinguishes lorlatinib from other acyclic ALK inhibitors, enhancing its metabolic stability and decreasing the predilection for high p-glycoprotein 1-mediated efflux out of the CNS. ${ }^{14}$ 
In an era of emerging therapies for ALK-positive NSCLC, our review examines the role of lorlatinib in reducing the risk of disease progression and its current place in therapy in patients with advanced ALK-positive NSCLC.

\section{Systemic Response of Lorlatinib in Advanced ALK-Positive NSCLC}

Significant clinical benefits have been observed with oral ALK tyrosine kinase inhibitors (TKIs) over chemotherapy for metastatic ALK-positive NSCLC. ${ }^{4,5}$ Crizotinib, alectinib, brigatinib, ceritinib, and lorlatinib are currently approved in the first-line setting for treatment of advanced ALK-positive lung cancers. ${ }^{4,5,7-9,11}$ Lorlatinib was developed to target mutants resistant to first- and second-generation ALK TKIs. Preclinical data using cell assays demonstrated the potency of lorlatinib in inhibiting ALK phosphorylation and cell growth in a variety of ALK-resistant mutations, including L1196M and G1269A, which are common crizotinib-resistant mutations, as well as G1202R, a common resistant mutation occurring with first- and second-generation ALK inhibitors. ${ }^{15}$ In the phase 2 clinical study, a systemic objective response (OR) was seen in $90 \%(95 \%$ CI, 73.5-97.9) of treatment-naïve patients with advanced ALK-positive NSCLC receiving lorlatinib and 32.1 to $69.5 \%$ in patients who received lorlatinib as subsequent line therapy following firstor second-generation ALK TKIs with or without chemotherapy. ${ }^{12}$ Results of the phase 2 trial illustrated the promising antitumor activity of lorlatinib both as first and subsequent line therapy.

Given the clinical benefits in response seen in phase 1 and preliminary phase 2 studies, lorlatinib received accelerated FDA approval for second- or third-line treatment of ALK-positive metastatic NSCLC in November 2018 and it was on the basis of the phase 3 data from the CROWN trial that approval of lorlatinib as a first-line therapy was given in March $2021{ }^{11,12}$ In the open label, phase 3, multicenter CROWN trial, patients with treatment-naïve advanced ALK-positive NSCLC were randomized to receive either lorlatinib at $100 \mathrm{mg}$ daily or crizotinib at $250 \mathrm{mg}$ twice daily. Systemic OR was $76 \%$ (95\% CI, 68 to 83 ) with lorlatinib versus $58 \%(95 \% \mathrm{CI}, 49$ to 66$)$ with crizotinib, including complete response in $3 \%$ and $0 \%$, respectively. Duration of response (DOR) was longer with lorlatinib, with $70 \%$ of patients who received lorlatinib and $27 \%$ of patients who received crizotinib having a response that lasted at least 12 months. Median progression-free survival (PFS), as determined by blinded independent central review (BICR), was not estimable in the lorlatinib arm and 9.3 months (95\% CI, 7.6 to 11.1) in the crizotinib arm. Although the overall survival (OS) data was not available at the time of PFS analysis, the hazard ratio (HR) for disease progression or death was $0.28 .^{11}$

At the time CROWN was initiated, crizotinib was the standard-of-care first-line treatment for metastatic ALKpositive NSCLC. Over the last several years, phase 3 clinical trials have shown superior efficacy of second-generation TKIs over crizotinib and chemotherapy in the first-line setting. ${ }^{8,9,16}$ However, no randomized controlled clinical trials have directly compared lorlatinib with second-generation ALK TKIs (Table 1). In the ALEX trial comparing alectinib with crizotinib, alectinib had a superior OR of $82.9 \%$ versus $75.5 \%$ for crizotinib with an HR for disease progression or death of $0.47 .{ }^{9}$ Similarly, in the ALTA-1L trial, brigatinib demonstrated an OR of $74 \%$ compared to $62 \%$ with crizotinib and an HR for disease progression or death of $0.48 .{ }^{17}$ Acknowledging the limitations of cross-trial comparisons, lorlatinib had a lower HR for disease progression or death of 0.28 relative to alectinib and brigatinib. ${ }^{11}$

One of the particular challenges barring comparisons in these cross-trial analyses is the different primary end points used in each trial. In ALEX, the primary end point was investigator-assessed PFS while the primary end point was PFS as determined by BICR in ALTA-1L and CROWN. Investigator-assessed PFS was a secondary end point in ALTA-1L and CROWN. Median PFS by independent central review was 25.7 and 24.0 months for alectinib and brigatinib, respectively, while BICR and investigator-assessed median PFS was not reached at the time of data analysis for lorlatinib in CROWN. ${ }^{9,11,17,18}$ Unlike alectinib, brigatinib, and lorlatinib, no head-to-head trials have compared outcomes between ceritinib and crizotinib in previously untreated advanced ALK-positive NSCLC. Ceritinib is among the first-line FDA approved therapies for treatment of advanced ALK-positive NSCLC after the results from the ASCEND-4 trial comparing ceritinib to standard-of-care chemotherapy. In the phase 3 ASCEND-4 study, the objective response rate (ORR) was $72.5 \%$. BICR and investigatorassessed median PFS was 16.6 and 16.8 months, respectively, for patients who received ceritinib. ${ }^{16}$

Clinical benefits of lorlatinib are seen not only in the first-line setting but also in patients who progress on previous second-generation ALK inhibitors. Of the patients treated with at least one prior second-generation ALK TKI, the OR rate was $39.6 \%$, median DOR was 9.6 months, median PFS was 6.6 months and median OS was 20.7 months. ${ }^{19}$ Efficacy of lorlatinib following a second-generation ALK TKI further differs according to ALK resistance 
Table I Systemic Response of Next-generation ALK Inhibitors

\begin{tabular}{|c|c|c|c|c|}
\hline & Alectinib & Brigatinib & Ceritinib & Lorlatinib \\
\hline Clinical trial & ALEX $^{9,18}$ & ALTA-IL ${ }^{17,22}$ & ASCEND-4 $4^{16}$ & CROWN"I \\
\hline OR (\%) $(95 \% \mathrm{Cl})$ & $82.9(76.0-88.5)$ & $74(66-8 I)$ & $72.5(65.5-78.7)$ & $76(68-83)$ \\
\hline Median DOR (months) $(95 \% \mathrm{Cl})$ & NE (NE) & $33.2(22.1-\mathrm{NE})$ & $23.9(16.6-N E)$ & NE (NE - NE) \\
\hline $\begin{array}{l}\text { Median PFS by ICR (months) } \\
(95 \% \mathrm{Cl})\end{array}$ & $25.7(19.9-\mathrm{NE})$ & $24.0(18.5-43.2)^{*}$ & $16.6(12.6-27.2)^{*}$ & NE (NE - NE)* \\
\hline Median PFS by IR (months) $(95 \% \mathrm{Cl})$ & $34.8(17.7-\mathrm{NE})^{*}$ & $30.8(21.3-40.6)$ & $16.8(13.5-25.2)$ & NE (NE - NE) \\
\hline $\begin{array}{l}\text { HR for disease progression or } \\
\text { death }(95 \% \mathrm{Cl})\end{array}$ & $0.47(0.34-0.65)$ & $0.48(0.35-0.66)$ & $0.55(0.42-0.73)$ & $0.28(0.19-0.41)$ \\
\hline OS rates $(\%)(95 \% \mathrm{Cl})$ & $\begin{array}{l}5 \text {-year OS rate } 62.5 \% \\
(54.3-70.8)\end{array}$ & $\begin{array}{l}3 \text { year-OS probability } \\
71 \%(62-78)\end{array}$ & $\begin{array}{l}2 \text { year-OS probability } 70.6 \% \\
(62.2-77.5)\end{array}$ & NA \\
\hline Median OS, HR (95\% Cl) & $0.67(0.46-0.98)$ & $0.81(0.53-1.22)$ & $0.73(0.50-1.08)$ & $0.72(0.4 I-1.25)$ \\
\hline
\end{tabular}

Note: *Primary end point of the study.

Abbreviations: ICR, independent central review; $\mathrm{Cl}$, confidence interval; DOR, duration of response; HR, hazard ratio; IR, investigator review; NA, no available data; NE, not estimable; OR, overall response; OS, overall survival; PFS, progression-free survival.

mutation status. ORR in patients with ALK resistance mutations found on either plasma or tissue testing was 62 to $69 \%$ versus 27 to $32 \%$ in mutation-negative patients. Whereas significant differences in median PFS were not observed using plasma genotyping, median PFS and DOR were longer in patients with ALK resistant mutations found on tumor genotyping with a median PFS of 11.0 months versus 5.4 months and DOR of 24.4 months versus 4.3 months in mutation-positive versus mutation-negative patients, respectively. ${ }^{20}$ Hence, future directions in ALK-positive NSCLC may involve routine tumor genotyping after progression on second-generation TKIs in determining ALK resistance mutation status and using this data to predict the efficacy of lorlatinib.

\section{Intracranial Response of Lorlatinib in Advanced ALK-Positive NSCLC}

A common site for metastasis and relapse in NSCLC is the brain with CNS metastasis at baseline in approximately 30 $40 \%$ of patients with advanced ALK-positive NSCLC. ${ }^{8-10}$ P-glycoprotein 1-mediated drug efflux is postulated to play a role in the poor CNS penetration by crizotinib since crizotinib is a substrate of the P-glycoprotein efflux pump. Unlike crizotinib and owing to its macrocyclic structure, lorlatinib does not have an affinity for the P-glycoprotein efflux pump and is therefore able to infiltrate the blood-brain barrier and accumulate in the CNS. ${ }^{13}$

One of the significant findings from CROWN underscores the antitumor activity of lorlatinib on CNS lesions. Compared to crizotinib, lorlatinib demonstrated an improved intracranial response of $66 \%$ versus $20 \%$ in patients with and without measurable brain metastases, including a complete intracranial response of $61 \%$ versus $15 \%$ (OR $=8.41$; $95 \%$ CI, 2.59 27.23). Among patients with measurable brain metastasis at baseline, an intracranial response was seen in $82 \%$ of patients in the lorlatinib arm versus $23 \%$ in the crizotinib arm $(\mathrm{OR}=16.83 ; 95 \% \mathrm{CI}, 1.95-163.23)$. Additionally, lorlatinib demonstrated a greater duration of intracranial response and time to CNS progression. Lorlatinib was associated with a durable intracranial response of at least 12 months in $72 \%$ of patients versus $0 \%$ of patients receiving crizotinib. At 12 months, $96 \%$ of patients on lorlatinib were without CNS progression versus $60 \%$ of patients on crizotinib. ${ }^{11}$ Overall, lorlatinib demonstrates intracranial efficacy comparable to alectinib, brigatinib and ceritinib (Table 2). Alectinib and brigatinib had a 59\% and $66 \%$ rate of intracranial response, respectively, in patients with any CNS disease baseline while both ALK TKIs had an approximate $80 \%$ rate of response in patients with measurable brain metastasis. ${ }^{9,17}$

Moreover, the CNS response of lorlatinib is not limited to its use in the first-line setting. Lorlatinib is effective against CNS metastasis even in patients pretreated with second-generation ALK TKIs. Intracranial response of lorlatinib was 
Table 2 Intracranial Response of Next-generation ALK Inhibitors

\begin{tabular}{|c|c|c|c|c|}
\hline & Alectinib & Brigatinib & Ceritinib & Lorlatinib \\
\hline Clinical trial & ALEX ${ }^{9}$ & ALTA-IL 17 & ASCEND-4 $4^{16}$ & CROWN"I \\
\hline \multicolumn{5}{|c|}{ Intracranial efficacy in patients with any baseline brain metastasis } \\
\hline OR (\%) $(95 \% \mathrm{Cl})$ & $59(46-71)$ & $66(5 I-79)$ & $46.3(32.6-60.4)$ & $66(49-80)$ \\
\hline Median DOR (months) $(95 \% \mathrm{Cl})$ & NE $(17.3-N E)$ & $27.1(16.9-42.8)$ & NA & NE (NE - NE) \\
\hline \multicolumn{5}{|c|}{ Intracranial efficacy in patients with measurable baseline brain metastasis } \\
\hline OR (\%) $(95 \% \mathrm{Cl})$ & $81(58-95)$ & $78(52-94)$ & $72.7(49.8-89.3)$ & $82(57-96)$ \\
\hline Median DOR (months) $(95 \% \mathrm{Cl})$ & $17.3(\mid 4.8-N E)$ & $27.9(5.7-\mathrm{NE})$ & $16.6(8.1-N E)$ & NE (NE - NE) \\
\hline
\end{tabular}

Abbreviations: $\mathrm{Cl}$, confidence interval; DOR, duration of response; NA, no available data; NE, not estimable; OR, overall response.

$66.7 \%$ in patients with one prior second-generation ALK TKI and 54.2\% in patients with at least two prior secondgeneration ALK TKIs. ${ }^{19}$

\section{Safety of Lorlatinib}

Lorlatinib has a safety profile unique from other ALK TKIs. Hypercholesterolemia (70\%), hypertriglyceridemia (64\%), edema (55\%), weight gain (38\%), peripheral neuropathy (34\%), and cognitive side effects $(21 \%)$ are among the most commonly reported adverse events. Drug reactions with lorlatinib are primarily mild to moderate, with grade 3-4 adverse events mostly attributable to hypertriglyceridemia, weight gain, hypercholesterolemia, and hypertension. ${ }^{11}$

Hyperlipidemia is a distinct adverse side effect of lorlatinib that is mainly controlled with lipid-lowering therapies and dose interruptions or modifications. Specific CYP450 enzymes can interfere with lorlatinib metabolism so lipid-lowering agents that do not interact with CYP450 are preferred such as rosuvastatin. ${ }^{21}$

Another category of drug reactions for lorlatinib includes CNS effects. Cognitive side effects such as memory impairment, disturbance in attention, and amnesia were reported in $21 \%$ of patients on lorlatinib while mood changes were reported in $16 \% .{ }^{11}$ In a pooled analysis of patients from phase 1 and 2 studies, the median time to onset of cognitive side effects was 53 days and generally grade 2 or less in severity, with $7.4 \%$ of these cases grade 3 and no grade 4 events were reported. Cognitive effects were in fact the most common adverse event associated with lorlatinib dose reductions or interruptions (6.4\%), with resolution of cognitive side effects in $66.7 \%$ of cases following dose modifications and a median time to resolution of 10 days. In patients rechallenged with the same dose of lorlatinib, CNS effects recurred in $21.2 \%$ of patients while $12.1 \%$ recurred at lower doses. Similar to cognitive effects, mood effects were generally grade 1 or 2, with $6.5 \%$ of cases grade 3 and no grade 4 events reported. Mood effects resolved in $66.7 \%$ of cases requiring dose modifications with a median time to resolution of 14 days and recurrences occurred in $33 \%$ of patients who restarted lorlatinib at reduced doses. ${ }^{21}$

Whereas second-generation ALK TKIs were largely associated with diarrhea, nausea and elevated liver enzymes, these adverse events occurred less frequently with lorlatinib, with a reported incidence of about $15-20 \%{ }^{9,11,16,22}$

\section{Acquired ALK Resistance to Lorlatinib}

ALK resistance differs across a spectrum of mutations, with $20 \%$ of patients on crizotinib acquiring ALK resistant mutations and $56 \%$ of patients on second-generation ALK inhibitors. ${ }^{23}$ Among patients previously treated with first- and/or second-generation ALK inhibitors, the most common ALK mutations seen on tumor genotyping were G1202R (40\%), F1174X (20\%), I1171X (13\%), and G1269A (13\%), all of which are sensitive to lorlatinib. In the subgroup of patients who formerly received crizotinib, the most common ALK mutations were G1269A, F1174X, and L1196M; while in patients who previously received one or more second-generation ALK TKIs, the leading ALK mutation was G1202R, detected in $55 \%$ of cases. ${ }^{20}$ 
Lorlatinib is a potent ALK/ROS1 inhibitor with activity against single and compound ALK resistant mutations. In in vitro studies, lorlatinib inhibits ALK phosphorylation in single ALK mutants including ALK G1202R and retains potency against compound mutants including ALK D1203N/E1210K and ALK D1203N/F1174C. ${ }^{23}$ However, as with other ALK inhibitors, acquired resistance to lorlatinib invariably occurs.

One mechanism of resistance is the emergence of on-target resistance by compound mutations. Using in vitro mutagenesis screening, Yoda et al identified only compound and no single ALK mutations conferring high levels of resistance to lorlatinib. Of the compound mutations found in vitro, ALK C1156Y/L1198F and G1202R/L1196M were detected in these patient samples. ${ }^{24}$ Additional resistance to lorlatinib have been found in patient samples, including ALK I1171S/G1269A, L1196M/D1203N, G1202R/F1174L, G1202R/L1196M and D1203N/I1171N mutations as well as off-target mutations in NF2. ${ }^{25-27}$ Of note, $\mathrm{C} 1156 \mathrm{Y} / \mathrm{L} 1198 \mathrm{~F}$ mutations in one study retained susceptibility to lorlatinib in engineered cell mutants and patient-derived cells, suggesting the presence of off-target mechanisms in driving lorlatinib resistance in patients with this specific compound mutation. ${ }^{26}$ Although ALK C1156Y/L1198F mutants are resistant to second-generation ALK inhibitors and lorlatinib, this compound mutation interestingly imparts resensitization to crizotinib. ${ }^{28}$ Conversely, resistance to lorlatinib is mildly increased in ALK G1202R/ F1174L compound mutants compared to single G1202R mutants. Nevertheless, increasing lorlatinib dosing to overcome this resistance may not be considered a feasible strategy clinically as this would increase the risk for drug toxicities. ${ }^{26}$

On the other hand, off-target resistance is ALK-independent, with mechanisms that include bypass signaling and changes in cell types. Bypass signaling through the activation of EGFR, MET, KIT, KRAS or AXL as well as transformations to other lineages such as small cell or through SRC activation have been reported. ${ }^{24,26,29}$ Mutations leading to the loss of function in NF2, a tumor suppressor in the regulation of the PI3K-AKT-mTOR pathway, were also found to confer resistance to lorlatinib, suggesting a potentially therapeutic role for mTOR inhibitors in patients harboring these mutations. ${ }^{26}$

Lorlatinib resistance poses a clinical challenge given the limited options for treatment once this occurs. Gilteritinib, a TKI approved for the treatment of FLT3-positive acute myeloid leukemia, has been found to inhibit tumor growth in EML4-ALK I1171N/F1174I compound mutants in vivo. ${ }^{29}$ A next-generation macrocyclic ALK inhibitor, TPX-0131, demonstrated anti-tumor activity in vivo in a range of single and compound mutants refractory to all approved ALK inhibitors and currently being studied in the phase 1 FORGE- 1 trial. ${ }^{30}$ With lorlatinib as the only third and latest generation of ALK TKIs for advanced ALK-positive NSCLC, additional studies are needed to find novel strategies to overcome its resistance upon progression.

\section{Discussion}

In this exciting era of personalized medicine, we have multiple targeted therapies approved for treatment of patients with advanced ALK-positive NSCLC. Lorlatinib joins alectinib, brigatinib, crizotinib, and ceritinib in the ranks of approved ALK TKIs for use in the first-line setting for metastatic ALK-positive lung cancers. Preclinical data demonstrated the potency of lorlatinib in inhibiting ALK phosphorylation and cell growth across various resistance mutations occurring with first- and second-generation ALK inhibitors. ${ }^{15}$ In clinical studies, CROWN showed the superior efficacy of lorlatinib over crizotinib in terms of systemic and intracranial response, with a statistically significant lower risk for disease progression or death with lorlatinib. ${ }^{11}$ CROWN is the only clinical study that has directly compared lorlatinib with another ALK inhibitor and in this case with a first-generation ALK TKI, crizotinib. In the absence of head-to-head trials with second-generation ALK inhibitors, we currently do not have data to show that the risk reduction in disease progression or death with lorlatinib exceeds other second-generation ALK TKIs.

Without randomized controlled clinical trials directly comparing lorlatinib with other second-generation ALK inhibitors, there is no data supporting the superiority of lorlatinib over second-generation therapies in the frontline setting. On the basis of cross-trial analyses, similar outcomes were seen with lorlatinib in CROWN and brigatinib in ALTA-1L with an approximate systemic OR of $75 \%$ and intracranial response of $80 \%$ in patients with measurable brain metastasis. OS was not reached with either lorlatinib or brigatinib in their respective phase 3 trials and neither with crizotinib at the final OS analysis in PROFILE 1014. ${ }^{11,12,17,22}$ In the absence of randomized studies, it therefore begs the 
question of where lorlatinib stands relative to other next-generation ALK TKIs in treatment-naïve patients with advanced ALK-positive NSCLC. A retrospective analysis estimated that the median OS from diagnosis of metastatic disease for patients receiving next-generation ALK inhibitors after crizotinib was 89.6 months (95\% CI, 53.5-NR), signifying the overall nonaggressive course of ALK-positive lung disease. ${ }^{31,32}$ Therefore, it is reasonable to reserve lorlatinib as a later line therapy with the prospect of overcoming a range of ALK resistance mechanisms generated by earlier-generation ALK TKIs since lorlatinib retains an OR of $40 \%$ in patients treated with at least one prior second-generation ALK TKI. ${ }^{19}$

Use of sequential therapy with ALK inhibitors was found to be effective in several case reports of patients with meningeal carcinomatosis and ALK-positive NSCLC resistant to early generations of ALK-inhibitors. ${ }^{33-35}$ One patient who developed meningeal carcinomatosis following initial therapy with alectinib and brigatinib experienced resolution of CNS symptoms and radiographic disease after starting lorlatinib with no disease progression at 8 months from lorlatinib initiation. ${ }^{35}$ Another case report of a patient with recurrent brain metastasis and meningeal carcinomatosis on second-line alectinib and intracranial disease refractory to ceritinib, brigatinib, and alectinib rechallenge responded to lorlatinib in the sixth-line setting, with near resolution of CNS lesions 4.1 months after starting lorlatinib. Time from the start of crizotinib to lorlatinib in this case was 89.5 months. ${ }^{33}$ Sequential ALK inhibitor therapy while utilizing lorlatinib in subsequent lines of treatment in these cases provides examples of the potential efficacy and improved survival that this treatment strategy may portend in patients with ALK-mutated NSCLC, particularly those patients who develop meningeal dissemination on second-generation ALK inhibitors.

Among the myriad of ALK targeted therapies, the risk for hyperlipidemia and cognitive and mood adverse effects are unique to lorlatinib. ${ }^{11}$ Hyperlipidemia is generally manageable with lipid-lowering agents or dose reductions in lorlatinib. CNS side effects including cognitive and mood effects are typically reversible with stopping or dose reducing lorlatinib and may recur with rechallenge at lower doses. ${ }^{21}$ Given these particular CNS effects with lorlatinib, patients with underlying psychiatric or cognitive disorders should be monitored closely on lorlatinib or receive an alternative ALK TKI.

Overall, lorlatinib exhibits potential antitumor activity in both the first- and subsequent-line setting. Unlike advanced EGFR-positive NSCLC where the strategy is to use osimertinib or our best drug upfront, a large proportion of patients with advanced ALK-positive NSCLC are expected to see second-line therapy as a result of the prolonged duration of response with early-generation ALK TKIs. As a whole, ALK TKIs represent a promising class of drugs in targeting advanced ALK-mutated NSCLC. Given the potency of lorlatinib against ALK-positive lung cancers, lorlatinib should be employed when initial second-generation ALK TKIs are no longer able to keep cancer at bay.

\section{Abbreviations}

ALK, anaplastic lymphoma kinase; BICR, blinded independent central review; CNS, central nervous system; FDA, Food and Drug Administration; NSCLC, non-small cell lung cancer; ROS1, c-ros oncogene 1; TKI, tyrosine kinase inhibitor.

\section{Funding}

This review was not supported by any external funding.

\section{Disclosure}

Karen M Yun has no competing interests. Lyudmila A Bazhenova reports personal fees from ORIC, Turning Point Therapeutics, Neuvogen, Daichi, BMS, Janssen, Merck, BeyondSpring, Regeneron, G1 Therapeutics, Bayer, AstraZeneca, Takeda, Blueprint, Boehringer Ingelheim, Novartis, and Genentech outside the submitted work.

\section{References}

1. Kwak EL, Shaw AT, Ou SHI, et al. Anaplastic lymphoma kinase inhibition in non-small-cell lung cancer. $N$ Engl J Med. 2010;11:1693-1703.

2. Soda M, Choi YL, Enomoto M, et al. Identification of the transforming EML4-ALK fusion gene in non-small-cell lung cancer. Nature. 2007;448 (7153):561-566. doi:10.1038/nature05945

3. Du X, Shao Y, Qin HF, Tai YH, Gao HJ. ALK-rearrangement in non-small-cell lung cancer (NSCLC). Thorac Cancer. 2018;9(4):423-430. doi:10.1111/1759-7714.12613 
4. Solomon BJ, Mok T, Kim DW, et al. First-line crizotinib versus chemotherapy in ALK-positive lung cancer. $N$ Engl J Med. 2014;371 (23):2167-2177. doi:10.1056/NEJMoa1408440

5. Shaw AT, Kim DW, Nakagawa K, et al. Crizotinib versus chemotherapy in advanced ALK-positive lung cancer. $N$ Engl J Med. 2013;368 (25):2385-2394. doi:10.1056/NEJMoa1214886

6. Costa DB, Shaw AT, Ou SHI, et al. Clinical experience with crizotinib in patients with advanced alk-rearranged non-small-cell lung cancer and brain metastases. J Clin Oncol. 2015;33(17):1881-1888. doi:10.1200/JCO.2014.59.0539

7. Shaw AT, Kim TM, Crinò L, et al. Ceritinib versus chemotherapy in patients with ALK-rearranged non-small-cell lung cancer previously given chemotherapy and crizotinib (ASCEND-5): a randomised, controlled, open-label, phase 3 trial. Lancet Oncol. 2017;18(7):874-886. doi:10.1016/ S1470-2045(17)30339-X

8. Camidge DR, Kim HR, Ahn MJ, et al. Brigatinib versus crizotinib in ALK-positive non-small-cell lung cancer. $N$ Engl $J$ Med. 2018;379:2027-2039. doi:10.1056/NEJMoa1810171

9. Peters S, Camidge DR, Shaw AT, et al. Alectinib versus crizotinib in untreated ALK-positive non-small-cell lung cancer. New Engl J Med. 2017;377:829-838. doi:10.1056/NEJMoa1704795

10. Horn L, Wang Z, Wu G, et al. Ensartinib vs crizotinib for patients with anaplastic lymphoma kinase-positive non-small cell lung cancer: a Randomized Clinical Trial. JAMA Oncol. 2021;7(11):1617. doi:10.1001/jamaoncol.2021.3523

11. Shaw AT, Bauer TM, de Marinis F, et al. First-line lorlatinib or crizotinib in advanced ALK-positive lung cancer. $N$ Engl J Med. 2020;383 (21):2018-2029. doi:10.1056/NEJMoa2027187

12. Solomon BJ, Besse B, Bauer TM, et al. Lorlatinib in patients with ALK-positive non-small-cell lung cancer: results from a Global Phase 2 Study. Lancet Oncol. 2018;19(12):1654-1667. doi:10.1016/S1470-2045(18)30649-1

13. Shaw AT, Felip E, Bauer TM, et al. Lorlatinib in ALK- or ROS1-rearranged non-small cell lung cancer: an international, multicenter, open-label phase 1 trial. Lancet Oncol. 2017;18(12):1590-1599. doi:10.1016/S1470-2045(17)30680-0

14. Johnson TW, Richardson PF, Bailey S, et al. Discovery of (10R)-7-Amino-12-fluoro-2,10,16-trimethyl-15-oxo-10,15,16,17-tetrahydro-2H8,4-(metheno)pyrazolo[4,3-h][2,5,11]-benzoxadiazacyclotetradecine-3-carbonitrile (PF-06463922), a Macrocyclic Inhibitor of Anaplastic Lymphoma Kinase (ALK) and c-ros Oncogene 1 (ROS1) with Preclinical Brain Exposure and Broad-Spectrum Potency against ALK-Resistant Mutations. J Med Chem. 2014;57(11):4720-4744. doi:10.1021/jm500261q

15. Zou HY, Friboulet L, Kodack DP, et al. PF-06463922, an ALK/ROS1 inhibitor, overcomes resistance to first and second generation ALK inhibitors in preclinical models. Cancer Cell. 2015;28(1):70-81. doi:10.1016/j.ccell.2015.05.010

16. Soria JC, Tan DSW, Chiari R, et al. First-line ceritinib versus platinum-based chemotherapy in advanced ALK-rearranged non-small-cell lung cancer (ASCEND-4): a randomised, open-label, phase 3 study. Lancet. 2017;389(10072):917-929. doi:10.1016/S0140-6736(17)30123-X

17. Camidge DR, Kim HR, Ahn MJ, et al. Brigatinib versus crizotinib in ALK inhibitor-naive advanced ALK-positive NSCLC: final results of phase 3 ALTA-1L trial. $J$ Thorac Oncol. 2021;16(12):2091-2108. doi:10.1016/j.jtho.2021.07.035

18. Mok T, Camidge DR, Gadgeel SM, et al. Updated overall survival and final progression-free survival data for patients with treatment-naive advanced ALK-positive non-small-cell lung cancer in the ALEX study. Ann Oncol. 2020;31(8):1056-1064. doi:10.1016/j.annonc.2020.04.478

19. Felip E, Shaw AT, Bearz A, et al. Intracranial and extracranial efficacy of lorlatinib in patients with ALK-positive non-small-cell lung cancer previously treated with second-generation ALK TKIs. Ann Oncol. 2021;32(5):620-630. doi:10.1016/j.annonc.2021.02.012

20. Shaw AT, Solomon BJ, Besse B, et al. ALK resistance mutations and efficacy of lorlatinib in advanced anaplastic lymphoma kinase-positive nonsmall-cell lung cancer. J Clin Oncol. 2019;37(16):1370-1379. doi:10.1200/JCO.18.02236

21. Bauer TM, Felip E, Solomon BJ, et al. Clinical management of adverse events associated with lorlatinib. Oncologist. 2019;24(8):1103-1110. doi:10.1634/theoncologist.2018-0380

22. Camidge DR, Kim HR, Ahn MJ, et al. Brigatinib versus crizotinib in advanced ALK inhibitor-naive ALK-positive non-small cell lung cancer: second interim analysis of the Phase III ALTA-1L trial. J Clin Oncol. 2020;38:3592-3603. doi:10.1200/JCO.20.00505

23. Gainor JF, Dardaei L, Yoda S, et al. Molecular mechanisms of resistance to first- and second-generation ALK inhibitors in ALK-rearranged lung cancer. Cancer Discov. 2016;6(10):1118-1133. doi:10.1158/2159-8290.CD-16-0596

24. Yoda S, Lin JJ, Lawrence MS, et al. Sequential alk inhibitors can select for lorlatinib-resistant compound ALK mutations in ALK-positive lung cancer. Cancer Discov. 2018;8(6):714-729. doi:10.1158/2159-8290.CD-17-1256

25. Dagogo-Jack I, Rooney M, Lin JJ, et al. Treatment with next-generation ALK inhibitors fuels plasma ALK mutation diversity. Clin Cancer Res. 2019;25(22):6662-6670. doi:10.1158/1078-0432.CCR-19-1436

26. Recondo G, Mezquita L, Facchinetti F, et al. Diverse resistance mechanisms to the third-generation ALK inhibitor lorlatinib in ALK-rearranged lung cancer. Clin Cancer Res off J Am Assoc Cancer Res. 2019;26(1):242-255. doi:10.1158/1078-0432.CCR-19-1104

27. Takahashi K, Seto Y, Okada K, et al. Overcoming resistance by ALK compound mutation (I1171S + G1269A) after sequential treatment of multiple ALK inhibitors in non-small cell lung cancer. Thorac Cancer. 2020;11(3):581-587. doi:10.1111/1759-7714.13299

28. Shaw AT, Friboulet L, Leshchiner I, et al. Resensitization to crizotinib by the lorlatinib ALK resistance mutation L1198F. N Engl J Med. 2016;374 (1):54-61. doi:10.1056/NEJMoa1508887

29. Mizuta H, Okada K, Araki M, et al. Gilteritinib overcomes lorlatinib resistance in ALK-rearranged cancer. Nat Commun. $2021 ; 12(1): 1261$. doi:10.1038/s41467-021-21396-w

30. Murray BW, Zhai D, Deng W, et al. Abstract 1469: TPX-0131, a potent inhibitor of wild type ALK and a broad spectrum of both single and compound ALK resistance mutations. Cancer Res. 2021;81(13Supplement):1469. doi:10.1158/1538-7445.AM2021-1469

31. Duruisseaux M, Besse B, Cadranel J, et al. Overall survival with crizotinib and next-generation ALK inhibitors in ALK-positive non-small-cell lung cancer (IFCT-1302 CLINALK): a French nationwide cohort retrospective study. Oncotarget. 2017;8(13):21903-21917. doi:10.18632/ oncotarget. 15746

32. Camidge DR. Lorlatinib should not be considered as the preferred first-line option in patients with advanced ALK rearranged NSCLC. $J$ Thorac Oncol. 2021;16(4):528-531. doi:10.1016/j.jtho.2020.12.022

33. Yamamoto K, Toyokawa G, Kozuma Y, Shoji F, Yamazaki K, Takeo S. ALK-positive lung cancer in a patient with recurrent brain metastases and meningeal dissemination who achieved long-term survival of more than seven years with sequential treatment of five ALK-inhibitors: a case report. Thorac Cancer. 2021;12(11):1761-1764. doi:10.1111/1759-7714.13962 
34. Li Z, Li P, Yan B, et al. Sequential ALK inhibitor treatment benefits patient with leptomeningeal metastasis harboring non-EML4-ALK rearrangements detected from cerebrospinal fluid: a case report. Thorac Cancer. 2020;11(1):176-180. doi:10.1111/1759-7714.13259

35. Nakashima K, Demura Y, Kurokawa K, et al. Successful treatment with lorlatinib in a patient with meningeal carcinomatosis of ALK-positive non-small cell lung cancer resistant to alectinib and brigatinib: a case report. Medicine. 2021;100(39):e27385. doi:10.1097/ MD.0000000000027385

\section{Publish your work in this journal}

Cancer Management and Research is an international, peer-reviewed open access journal focusing on cancer research and the optimal use of preventative and integrated treatment interventions to achieve improved outcomes, enhanced survival and quality of life for the cancer patient. The manuscript management system is completely online and includes a very quick and fair peer-review system, which is all easy to use. Visit http://www.dovepress.com/testimonials.php to read real quotes from published authors.

Submit your manuscript here: https://www.dovepress.com/cancer-management-and-research-journal 